\title{
Sobre a botânica, a etnofarmacologia e a química de Calycophyllum spruceanum (Benth.) Hook. f. ex K. Schum
}

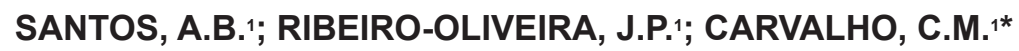

1Universidade Federal do Acre, Centro de Ciências Biológicas e da Natureza, 69.920-900, Rio Branco-AC, Brasil;

*Autor para correspondência: claricemaiacarvalho@gmail.com

\begin{abstract}
RESUMO: O fato de possuir espécies com potencial econômico ilimitado, faz com que o ambiente amazônico se torne alvo constante da biopirataria e da extração predatória dos recursos. Muitas espécies deste ambiente sequer possuem catalogação e, mesmo assim, encontram risco eminente de desaparecimento. Neste âmbito, trabalhos que reúnam dados sobre estas espécies possuem grande valor científico, cultural e econômico, sendo este o estímulo que desencadeou a produção desta revisão. Aqui a espécie Calycophyllum spruceanum (Benth.) Hook. f. ex K. Schum., uma Rubiaceae amazônica, é contemplada por meio de características botânicas, indicações etnofarmacológicas e propriedades químicas. Popularmente conhecida como mulateiro, a espécie é frequentemente receitada na etnomedicina como cicatrizante e rejuvenescedor, além de ser usada no controle de manchas de pele. Como peculiaridade botânica, mulateiro apresenta tronco retilíneo com epiderme fina esverdeada que evolui para uma periderme castanho-escuro, que é anualmente renovada. Em termos químicos, há destaque para a presença de alcalóides, taninos e, sobretudo, secoiridóides (7-metoxididerrosideo, 6'-acetil- $\beta$-D-glucopiranosildiderrosideo e 8-0-tigloildiderrosideo são peculiares à espécie). Mesmo com propriedades fotoprotetoras comprovadas, $C$. spruceanum ainda carece de pesquisas, sobretudo àquelas voltadas para a produção ex situ da planta $e$ àquelas que demonstrem a relação entre a ecologia da planta e a produção de metabólitos funcionais para a indústria.
\end{abstract}

Palavras-chaves: Rubiaceae, mulateiro, extração predatória.

\begin{abstract}
On the botany, ethnopharmacology, and chemistry of Calycophyllum spruceanum (Benth.) Hook. f. ex K. Schum. The Amazonian environment is a constant target for biopiracy and predatory extraction of resources due to the species with vast economic potential. Many of these species have not even been cataloged, and yet are already at imminent risk of extinction. In this paper, Calycophyllum spruceanum (Benth.) Hook. f. ex k. Schum., an Amazonian Rubiaceae, is studied with information on its botanical characteristics, ethnopharmacological uses, and chemical properties. Popularly known as mulateiro, the species is often prescribed in ethnomedicine for healing and vitality, and is also used to control skin patches. As a botanical peculiarity, it has a thin green epidermis that evolves into a dark brown periderm, which is renewed annually. In terms of chemistry, there is emphasis on the presence of alkaloids, tannins, and, especially, secoiridoids (7-methoxydiderroside, 6'-acetyl$\beta$-D-glucopyranosyldiderroside, and 8-O-tigloyldiderroside are peculiar to the species). Even with proven photoprotective properties, research on C. spruceanum is still lacking, in particular studies aimed at the ex situ production of the plant and those that show the relationship between the plant's ecology and the production of functional metabolites for the industry.
\end{abstract}

Keywords: Rubiaceae, mulateiro, predatory extraction.

\section{INTRODUÇÃO}

Em termos gerais, há grande carência de trabalhos que demonstrem o aglomerado de informações gerado ao longo dos anos sobre as espécies nativas do Brasil (Ribeiro-Oliveira \& Ranal, 2014). Isto possivelmente se deve a inserção de livros que contemplem aspectos gerais destas 
espécies. Entretanto, a atualização desses livros nem sempre é anual. Assim, trabalhos que reúnam o compilado da literatura são de grande valia para dar suporte à nova geração de pesquisadores. Em se tratando de espécies amazônicas isto é ainda mais significativo, visto a grande pulverização da informação em detrimento da potencialidade econômica destas espécies, que são foco de pesquisas farmacológicas, ecológicas e botânicas. Um exemplo disto é Calycophyllum spruceanum (Benth.) Hook. f. ex K. Schum.

Calycophyllum spruceanum é uma espécie natural da região amazônica, conhecida no Brasil como pau-mulato, pau-mulato-de-várzea, escorregamacaco e pau-marfim (Record \& Hess, 1943; Rizzini, 1971; Guitton, 1991); e, no Peru, como capirona, capirona de bajo ou capirona negra (Ugarte-Guerra \& Domínguez-Torrejón, 2010). Trata-se de uma árvore com madeira densa $\left(0,78 \mathrm{~g} \mathrm{~cm}^{-3}\right)$, muito utilizada na produção de cabo para ferramentas, molduras, pisos e esquadrias. Isto porque esta madeira, com elevada flexibilidade e facilidade para secagem ao ar, possui resistência moderada à deterioração, além de apresentar características que a enquadram como de boa manipulação (serragem, aplainagem, faqueagem, desenrolagem, torneagem, colagem, parafusagem e pregagem), evernizagem, pintura e emassamento (Guitton, 1991; Lorenzi, 1998; Ugarte-Guerra \& Domínguez-Torrejón, 2010). Pela baixa exigência em fertilidade dos solos, $C$. spruceanum também costuma ser empregada em plantios de áreas ciliares degradadas (Lorenzi, 1998; Maranho et al., 2013), sendo considerada uma boa alternativa para a recuperação deste tipo de área (Maranho et al., 2013).

Além desses usos convencionais, Calycophyllum spruceanum possui grande apelo etnofarmacológico (vide tópico Etnofarmacologia). Isto, aliado a produção de metabólitos funcionais para o tratamento de doenças (vide tópico Metabólitos), deixa evidente o potencial farmacológico da espécie. Apesar disto, não há compilado na literatura sobre o C. spruceanum. Por isto, este trabalho explana sobre os aspectos botânicos, etnofarmacológicos e propriedades químicas desta espécie a fim de subsidiar e estimular a nova geração de pesquisadores, sobretudo os que trabalham com espécies amazônicas, a elaborar hipóteses inéditas e bem embasadas sobre a espécie.

\section{MATERIAL E MÉTODOS}

As bases de dados utilizadas na busca por trabalhos relacionados à Calycophyllum spruceanum foram Scholar Google, Science Direct, Pubmed, Web of knowledge e Periódicos da CAPES. As palavras-chave utilizadas foram Calycophyllum spruceanum, mulateiro, Calycophyllum, Rubiaceae, medicinal plant, ethnopharmacology, etnomedicine, metabolics, metabolite, botanic aspects, botanical aspects e/ou phytochemistry. O período de busca compreendeu os anos entre $1945-2015$.

\section{Caracterização botânica Família e gênero}

Rubiaceae é uma grande família da ordem das Rubiales, que consiste em 611 gêneros, com mais de 13.100 espécies entre ervas (lianas), arbustos e árvores, distribuídas principalmente em áreas tropicais (Govaerts et al., 2007). No Brasil, inclui mais de 2.000 espécies, em aproximadamente 120 gêneros (Tavares, 2013). Os membros da família têm folhas disposta em espiral ou opostas entre si, com estípulas. Em espécies de clima tropical, estas folhas geralmente são grandes e verdes; em de clima temperado, caducas; e de clima árido, na forma de agulhas. Espécies desse gênero podem ter uma ou muitas flores pequenas agrupadas (Pelikan, 1979).

Afamília Rubiaceae foi dividida em quatro subfamílias (Cinchonoideae, Ixoroideae, Antirheoideae e Rubioideae), sendo Calycophyllum spruceanum pertencente à Cinchonoideae (Robbrecht, 1988; Gemin, 2011). Os gêneros desta sub-família possuem perfil químico caracterizado pela presença de alcalóides, iridóides e antraquinonas (Young, 1996). Os alcalóides, aliás, são considerados marcadores químicos taxonômicos dessa subfamília, sendo também as moléculas químicas mais estudadas (Cardoso et al., 2008).

Além de Calycophyllum spruceanum, o gênero Calycophyllum possui outras três espécies (C. multiflorum Griseb., C. acreanum Ducke e C. obovatum (Ducke) Ducke), também chamadas popularmente de pau-mulato, mas com diferenças morfológicas e de local de ocorrência. C. multiflorum é uma árvore que atinge entre 10 e $25 \mathrm{~m}$ de altura, apresenta folhas pequenas $(5-8 \mathrm{~cm}$ de comprimento e 3-4 cm de largura) e ocorre no sul da América do Sul, especialmente na Bolívia, Paraguai, Argentina e Brasil, onde é encontrada nos estados de Mato Grosso e de Mato Grosso do Sul. C. acreanum, possui folhas grandes (acima de $20 \mathrm{~cm}$ de comprimento), brácteas minutíssimas, que envolvem as jovens cimeiras nas inflorescências, cálice com sépalas de tamanho semelhante e ocorre em matas de terra firme. C. obovatum, por sua vez, apresenta uma das sépalas inúmeras vezes maior que as demais, com formato obovado, estreitada na base e foliácea (Almeida, 2004).

\section{Calycophyllum spruceanum}

Esta arbórea atinge 20 a $35 \mathrm{~m}$ de altura, com 0,7 a $1,8 \mathrm{~m}$ de diâmetro à altura do peito $\mathrm{e}$

Rev. Bras. PI. Med., Campinas, v.18, n.1, supl. I, p.383-389, 2016. 
diâmetro da copa entre 4 e $5 \mathrm{~m}$. O ápice é agudo ou obtuso, com base pouco atenuada (Record \& Hess, 1943; Lorenzi, 1998). O tronco retilíneo (Figura 1) é esguio, ramificado apenas no ápice, com casca fina de coloração esverdeada quando nova (epiderme), a qual se torna marrom ou castanho-escura quando madura (periderme). Este tronco, que rebrota com facilidade, "descasca" anualmente em longas tiras, deixando exposta a camada interna avermelhada de textura lisa, com aparência envernizada (Record \& Hess, 1943). A coloração da madeira pode estar associada às características genéticas de populações de Calycophyllum spruceanum (Sotelo-Montes et al., 2007; Montes et al., 2008). Por isto, a fase juvenil dos indivíduos tem sido utilizada pelo melhoramento genético com finalidade madeireira (Weber \& Montes, 2005; Weber et al., 2009). Em geral, a madeira destes indivíduos é relativamente forte, com variação quanto a propriedades mecânicas em termos de linhagem (Montes \& Hernandez, 2007).

Em linhas gerais, o xilema da espécie possui características morfológicas compatíveis às encontradas em plantas da Família Rubiaceae. Essa estrutura é constituída por anéis de crescimento distintos, vasos arredondados de paredes espessas, elementos vasculares médios, placas de perfuração simples, providos de apêndices em uma ou ambas extremidades, ausência de parênquima axial, com raios heterogêneos e de dois tamanhos distintos, e fibras libriformes septadas (Baldin et al., 2014).

A planta é perenifólia e higrófita, com adaptabilidade a luminosidade ainda pouco esclarecida (não há consenso se a espécies é heliófita ou esciófita) (Record \& Hess, 1943; Rizzini, 1971; Lorenzi, 1998; Ugarte-Guerra \& Domínguez-
Torrejón, 2010). As folhas são simples, opostas, glabras, subcoriáceas, oblongas ou ovado-oblongas, pecioladas e peninérveas, medindo $9 \mathrm{a} 17 \mathrm{~cm}$ de comprimento e 6 a $7 \mathrm{~cm}$ de largura. Apresenta inflorescência terminais cimosas, sendo as flores agrupadas em cimeiras triflorais envolvidas (Figura 1), quando em botão, numa bráctea foliácea, sendo aromáticas, branco-esverdeadas e hermafroditas, medindo 10-12 mm de comprimento (Rizzini, 1971; Guitton, 1991; Lorenzi, 1998). O fruto, medindo entre 8 e $10 \mathrm{~mm}$ de comprimento, é do tipo cápsula oblonga, deiscente, com tricomas esparsos e duas valvas, possuindo endosperma carnoso (Rizzini, 1971; Revilla, 2001). O florescimento se dá entre os meses de junho e julho, com frutificação ocorrendo entre outubro e novembro (Lorenzi, 1998; Almeida, 2003).

O número de trabalhos sobre a produção ex situ da espécie é muito limitado. As sementes de cor parda escura são aladas, muito pequenas e dispersas pelo vento. Aárea de dispersão é bastante ampla, ao longo de todo o rio Amazonas, onde as plântulas se estabelecem principalmente sobre solos argilosos, férteis e inundáveis (Record \& Hess, 1943; Ugarte-Guerra \& Domínguez-Torrejón, 2010). Entretanto, nenhuma evidência foi obtida sobre a dispersão unidirecional das sementes por meio da água, o que desempenharia importante papel na determinação da estrutura genética de populações de Calycophyllum spruceanum em grande amplitude geográfica (Russell et al., 1999). A germinação das sementes ocorre 20 a 40 dias após a semeadura, mas com baixa germinabilidade.

Em se tratando de propagação assexual, estacas de caule submetidas a 3.000 ppm de ácido indolbutírico (AIB) apresentam eleva porcentagem

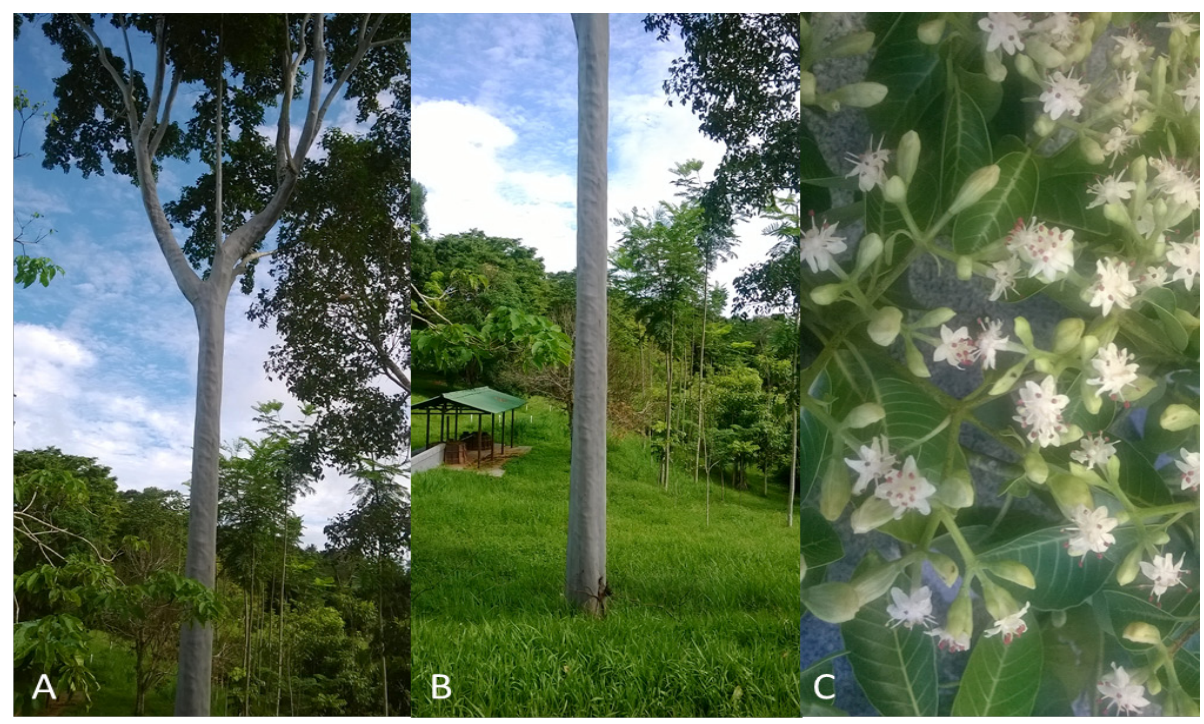

FIGURA 1. Árvore de Calycophyllum spruceanum (A). Detalhe do tronco retilíneo da espécie (B). Inflorescência de Calycophyllum spruceanum (C).

Rev. Bras. PI. Med., Campinas, v.18, n.1, supl. I, p.383-389, 2016. 
de enraizamento em curto período de tempo, i.e., doze dias após o plantio (Vallejos-Torres et al., 2014). Contudo, o crescimento e desenvolvimento inicial é lento e desuniforme (Gatti, 2002; MoraesNeto et al., 2003; Tauchen et al., 2011), com velocidade positivamente relacionada à dureza da madeira (Montes et al., 2007; Montes et al., 2008). As mudas requerem entre 6 e 8 meses para ser disponibilizadas para o plantio em local definitivo (Maranho, 2013). Devido a este comportamento de desenvolvimento (lento) há dificuldades de produção de mudas a partir de sementes, ainda que haja grande disseminação destas pelo vento (Gatti, 2002).

\section{Ocorrência da espécie}

Calycophyllum spruceanum ocorre em toda a Região Amazônica, abrangendo Bolívia, Brasil, Colômbia, Equador e Peru (Record \& Hess, 1943; Rizzini, 1971, Ugarte-Guerra \& Domínguez-Torrejón, 2010). É encontrada especialmente ao longo do rio Amazonas, onde forma agrupamentos quase homogêneos, chamados matas-de-pau-mulato (Brasil) ou capironais (Peru) (Lorenzi, 1998; UgarteGuerra \& Domínguez-Torrejón, 2010).

O estabelecimento da espécie parece estar associado ao tipo de solo. Neste âmbito, a espécie tem preferência por solos argilosos e arenosos, com conteúdo orgânico de médio a alto, $\mathrm{pH} 7$ e saturação de alumínio menor que $30 \%$. Não se desenvolve em solos muito ácidos, com pH entre 4 e 4,5 (Revilla, 2001).

Em contrapartida, é certo que a ocorrência está, em grande parte, condicionada à dinâmica dos rios, sendo frequentemente encontrada em praias de várzeas e em clareiras de matas de solos argilosos (Ducke \& Black, 1954; Lorenzi, 1998; Almeida, 2003). No Brasil, a espécie está praticamente restrita à Região Amazônica, sendo bastante difundida no Alto Amazonas, ao longo do rio Amazonas e no Alto Envira/Tarauacá, no Estado do Acre (Guitton, 1991; Lorenzi, 1998; Almeida, 2003).

\section{Etnofarmacologia}

Diferentes órgãos de Calycophyllum spruceanum são utilizados na medicina empírica. No Peru, o decocto e as infusões do córtex são empregados no tratamento de infecções da pele ou da mucosa oral (Carhuapona \& Ângulo, 1999); pastas da casca são utilizadas no tratamento de infecções oculares e no tratamento tópico de celulite (Revilla, 2001). Neste país, o decocto da casca da árvore também é usado no tratamento da "sarna negra", doença causada pelo ácaro Demodex canis, que vive sob a pele de cachorro (Shultes \& Raffauf, 1990). No Paraguai, é utilizada para tratar diabetes; e, na Colômbia, no controle de parasitas e doenças da pele (Revilla, 2001). No Brasil, a casca é utilizada na forma de chá como controle de manchas da pele e na prevenção ao envelhecimento (Caetano et al., 2014).

De maneira geral, a casca de Calycophyllum spruceanum é usada na forma de cataplasma, no tratamento de inflamações e de infecções fúngicas. Esta estrutura também é utilizada no preparo de emplastos capazes de curar cortes, feridas e queimaduras. Do córtex é feita a infusão, usada em infecções oculares, diabetes e males do ovário, sendo também utilizado no controle de doenças gástricas e uterinas (Almeida, 2003). Além disto, em um levantamento sobre o uso de plantas medicinais em comunidades de várzea dos rios Solimões e Amazonas, C. spruceanum foi citado como tratamento curativo de distúrbios do aparelho geniturinário, de inflamações, de colesterol alto e de problemas de tireóide, sendo a casca usada para fazer decocto ou para banhos de imersão (Cassino, 2010). Na cosmetologia, é utilizado na preparação de xampu para o controle da queda de cabelo, bem como no preparo de cremes e hidratantes curativos de rugas, manchas de pele e cicatrizes (Revilla, 2001; Araújo et al., 2007; Lino et al., 2009; Morais et al., 2009).

\section{Metabólitos}

Os metabólitos produzidos por Callycophyllum spruceanum são pouco estudados. Contudo, taninos e fenóis são bem relatados. Estes metabólitos parecem ser os responsáveis pelo desempenho da planta no retardo do envelhecimento celular e na fotoproteção por ações antioxidantes (Araújo et al., 2007; Lino et al., 2009). Aos taninos também é creditada a produção de ácido gálico, de cor marrom, que é empregado na indústria de tecido e couro (Emery et al., 2010).

A época de colheita (estiagem ou chuvosa) da casca (epiderme e/ou periderme) está diretamente relacionada a quantidade de taninos e polifenóis produzida (Costa et al., 2011). Do mesmo modo, o teor de cinza e o extrato apresentam diferenças quanto à época de colheita, sendo que o rendimento de cinzas na estiagem é $40,52 \%$ maior que no período chuvoso (Costa et al., 2011).

Extratos aquosos de Callycophyllum spruceanum foram estudados quanto ao método de preparo da solução de extração (decocto ou infusão - fator A), o tempo de extração (5 ou 15 minutos fator B) e a relação droga/solvente (fator $C$ ), a fim de estabelecer o teor de tanino total e o resíduo seco. Os resultados demonstraram que o método de extração, correspondente a interação do fator A e C, proporciona maior teor de tanino $(9,9 g \%)$ e resíduo seco $(0,88 \mathrm{~g} \%)$, sendo que há a possibilidade de outras substâncias ativas de interesse terem sido

Rev. Bras. PI. Med., Campinas, v.18, n.1, supl. I, p.383-389, 2016. 
negligenciadas por limitações técnicas (Costa et al., 2011), deflagrando a necessidade de mais estudos sobre métodos para a extração de metabólitos da espécie.

Zuleta et al. (2003) detalharam três novos secoiridóides presentes nos extratos etanólicos da casca de Callycophyllum spruceanum (7-metoxididerrosideo, 6'-acetil- $\beta$-Dglucopiranosildiderrosideo e 8-0-tigloildiderrosideo, Figura 2). Além destes, os autores relatam a ocorrência de outros iridóides previamente relacionados à espécie (loganetina, loganina, secoxiloganina e diderrosideo). Esses compostos poderão contribuir, por exemplo, na quimiotaxonomia de C. spruceanum, por meio do estabelecimento de limites entre os diversos gêneros encontrados na família Rubiaceae, considerada extremamente complexa.

\section{Atividade biológica}

Como mencionado anteriormente, Calycophyllum spruceanum possui metabólitos eficazes na proteção contra raios ultravioleta (UV), funcionando como rejuvenescedor. Lino et al. (2009), comprovaram as atividades antioxidantes e fotoprotetores dessa planta por meio de estudo com extratos aquosos e etanólicos das cascas. Este estudo foi conduzido com o teste de inibição da oxidação da micela mediada e os métodos 2,2-difenil-1-picrilhidrazil (DPPH) e 2,2'-azino-bis (3-etilbenzotiazolina) 6-ácido sulfônico (ABTS), ambos convencionalmente utilizados em ensaios de captura de radicais livres. No método DPPH, soluções com concentração de $357,14 \mu \mathrm{g} \mathrm{mL}^{-1}$ demonstraram que o extrato etanólico possui atividade $11,7 \%$ superior ao extrato aquoso $(73,8 \mathrm{e}$ $62,1 \%$ de atividade antioxidante, respectivamente). No ensaio ABTS, esta tendência se manteve, com o extrato etanólico apresentando atividade protetora $8,3 \%$ superior a do aquoso (respectivamente, $95,4 \mathrm{e}$ $87,1 \%$ de atividade nas concentração de $178,57 \mu \mathrm{g}$ $\mathrm{mL}^{-1}$ ). Assim, ficou comprovado que, embora ambos os extratos permitem a expressão da atividade antioxidante, os extratos alcoólicos apresentam melhor desempenho de extração de composto antioxidantes. No teste de inibição da oxidação da micela, mediada pela radiação UV, o extrato aquoso foi o mais ativo, protegendo a oxidação dos lipídeos em $96,5 \%$, na concentração de $2 \mathrm{mg} \mathrm{mL}^{-1}$, ratificando o potencial da planta para uso dermatológico.

Analisando a atividade contra formas tripomastigotas de Trypanosoma cruzi in vitro, Zuleta et al. (2003) observaram que os secoiridóides secoxiloganino e diderrosideo isolados do extrato etanólico da casca de Calycophyllum. spruceanum possuem atividade antitripanosoma. Outras

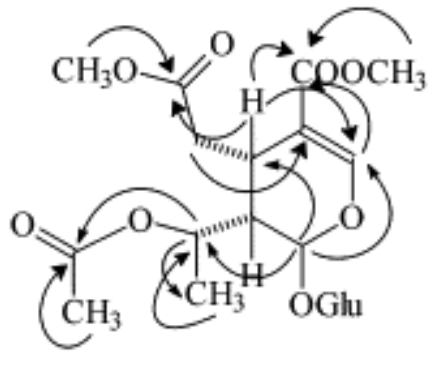

1

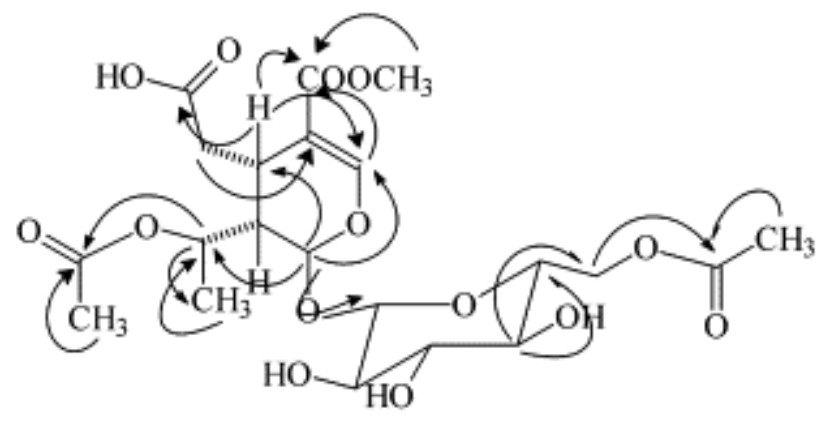

2

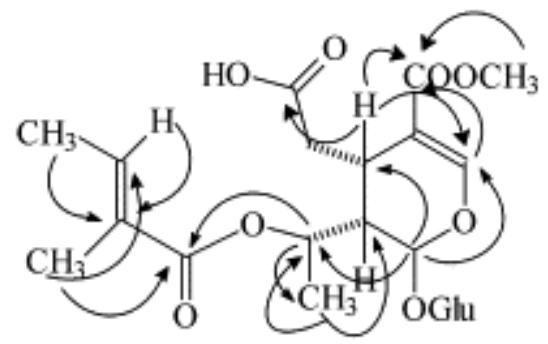

3

FIGURA 2. Secoiridóides presentes nos extratos etanólicos da casca de Callycophyllum spruceanum (1: 7-metoxididerrosideo; 2: 6'-acetil- $\beta$-D-glucopiranosildiderrosideo; e 3: 8-0-tigloildiderrosideo. Setas indicam correlações em Heteronuclear Multiple Bond Correlation (HMBC) entre moléculas de carbono e $\mathrm{H}-8$ em $\delta=$ 5,09. Fonte: Zuleta et al. (2003). 
espécies vegetais também apresentaram atividade biológica relacionada à secoiridóides. Portanto, a descoberta desses compostos também em $C$. spruceanum abre oportunidades para outros testes de atividade biológica de sucesso.

\section{CONSIDERAÇÕES}

O número escasso de trabalhos acerca dos aspectos químicos e farmacológicos de Callycophyllum spruceanum é evidente. Não obstante, há grande carência de estudos capazes de comprovarem a atividade biológica apontada pelo uso popular, evidenciando a necessidade de aprofundamento científico sobre o uso da espécie. Em vertente complementar, a espécie tem sido pobremente estudada em termos ecológicos, bem como pouco se discute a respeito do extrativismo predatório em detrimento ao interesse da indústria de cosméticos. Neste aspecto, esta revisão também deflagra a necessidade de mais estudos que apontem aspectos de cultivo ex situ da espécie, praticamente não encontrados na literatura e necessários para a produção em larga escala. A partir destes estudos, em certo, haverá redução da degradação do ambiente e da depressão genética. Aqui é interessante pontuar a necessidade de estudos que relacionem a produção de metabólitos vegetais com as diferentes condições de colheita do material vegetal, época de colheita e local de estabelecimento e/ou de cultivo das matrizes, sobretudo no que tange questões edafoclimáticas. $A$ superação destas barreiras pode proporcionar maior aproveitamento biotecnológico de Callycophyllum spruceanum, bem como resguardar a espécie da extinção eminente, acarretada pela exploração predatória.

\section{AGRADECIMENTOS}

Os autores são gratos ao Programa de Pós-graduação em Ciências Inovação e Tecnologia para a Amazônia (CITA), à Universidade Federal do Acre (UFAC), à CAPES, pela concessão da bolsa do segundo autor e à Fundação de Amparo à Pesquisa no Acre (FAPAC).

\section{REFERÊNCIAS}

ALMEIDA, M.C. Aspectos ecofisiológicos da germinação de sementes de multeiro (Calycophyllum spruceanum Benth.) - Rubiaceae. 2003. 116f. Tese (Doutorado em Ciências Biológicas) - Universidade Estadual Paulista, Rio Claro - SP.

ALMEIDA, M.C. Pau-mulato-da-várzea Calycophyllum spruceanum (Benth.) Hook. f. ex K. Schum. Informativo técnico rede de sementes da Amazônia, v. 6, [s.n.], p.01-02, 2004.

ARAÚJO, V.F. et al. Mulateiro. In: PASTORE JÚNIOR, F. et al. Plantas da Amazônia para a Produção Cosmética. 1.ed. Brasília: Editora Universidade de Brasília, 2007, p. 42-44.

BALDIN, T. et al. Análise preliminar do xilema secundário de Calycophyllum spruceanum (Benth) Hook. f. ex Schum (RUBIACEAE). In: SIMPÓSIO BRASILEIRO DE PÓS-GRADUAÇÃO EM CIÊNCIAS FLORESTAIS, 8, 2014, Recife. Anais Eletrônicos... Recife: UFRPE, 2014. p. 397-400.

CAETANO, R.S. et al. O uso de plantas medicinais utilizadas por frequentadores dos ambulatórios Santa Marcelina, RO. Revista Saúde e Pesquisa, v. 7, n. 1, p. 55-63, 2014.

CARDOSO, C.L. et al. Indole monoterpene alkaloids from Chimarrhis turbinata DC Prodr: a contribution to the chemotaxonomic studies of the Rubiaceae Family. Revista Brasileira de Farmacognosia, v.18, n. 1, p. 26-29, 2008.

CARHUAPONA, M.; ÂNGULO, P. Plantas Medicinales em Atencion Primaria de salud, Agroindrustria, Fitoquímica y Ecoturismo: perspectivas de desarrolho em laregionlos libertadores Wari. 1.ed. Lima: IICAGTZ,1999. 278p.

CASSINO, M.F. Estudo etnobotânico de plantas medicinais em comunidades de várzea do rio Solimões, Amazonas e aspectos farmacognósticos de Justicia pecroralis Jacq. forma mutuquinha (Acanthaceaea). 2010. 147f. Dissertação (Mestrado em Botânica) - Instituto Nacional de Pesquisas da Amazônia/Universidade Federal do Amazonas, Manaus.

COSTA, L. M. et al. Technological development of aqueous extracts from Calycophyllum spruceanum (mulateiro) using factorial design. Brazilian Journal of Pharmacognosy, v. 21, n. 1, p. 181-186, 2011.

DUCKE, A.; BLACK, E.A. Notas sobre Filogeografia da Amazônia Brasileira. Boletim técnico do Instituto Agronômico do Norte, v.29, [s.n.], p. 62, 1954.

GATTI, K.C. Propagação Vegetativa de Pau-Mulato (Calycophyllumspruceanum (Benth) K. Schum.), Jequitibá (Carinianaestrellensis (Raddi) Kuntze) e Teca (TectonagrandisLinn. F.) por miniestaquia. 2002. 83f. Tese (Doutorado em Ciência Florestal) Universidade Federal de Viçosa, Viçosa.

GOVAERTS, R., et al. World Checklist of Rubiaceae. The Board of Trustees of the Royal Botanic Gardens. 2007. Disponível em: http://www.kew.org/wcsp/ rubiaceae/ Acessado em 23 de julho de 2015.

GUITTON, T.L. Madeiras da Amazônia: características e utilização. 1.ed., Rio Branco: CEAGAC, 1991. 138 p.

LINO, T.S.S. et al. Efeito antioxidante e fotoprotetor de extratos aquosos e etanólicos da casca do Calycophyllum spruceanum. In: Reunião Anual da SBPC - Sociedade Brasileira para o Progresso da Ciência, 61., 2009, Manaus. Anais Eletrônicos... Manaus: UFAM, 2009.

LORENZI, H. Árvores brasileiras: Manual de identificação e cultivo de plantas arbóreas nativas do Brasil. 1.ed. Nova Odessa: Plantarum,1998. 317p.

MARANHO, A. et al. Crescimento inicial de espécies nativas com potencial madeireiro na Amazônia. Revista Árvore, v. 37, n. 5, p. 913-921, 2013. 
MONTES, C.S. et al. Genetic variation in wood mechanical properties of Calycophyllum spruceanum at an early age in the Peruvian Amazon. Wood and Fiber Science, v. 39, n. 4, p. 578-590, 2007.

MONTES, C.S. et al. Genetic variation in wood color and its correlations with tree growth and wood density of Calycophyllum spruceanum at an early age in the Peruvian Amazon. New Forests, v. 35, n. 1, p. 57-73, 2008.

MORAES NETO, S.P.D. et al. Fertilização de mudas de espécies arbóreas nativas e exóticas. Revista Árvore, v.27, n.2, p.129-137, 2003.

MORAIS, S.M. et al. Ação antioxidante de chás e condimentos de grande consumo no Brasil. Revista Brasileira de Farmacognosia, v. 19, n. 1, p. 315-320, 2009.

PELIKAN, W. The Rubiaceae. British Homoeopathic Journal, v. 68, n. 4, p.188-197, 1979.

RECORD, S.J.; HESS, R.W. Timbers of the New World. 1.ed. New Haven: Yale University Press, 1943. 643p.

REVILLA, J. Plantas da Amazônia: oportunidades econômicas e sustentáveis. 1.ed. Manaus: Sebrae/ INPA, 2001. 405p.

RIBEIRO-OLIVEIRA, J.P.; RANAL, M.A. Sementes florestais brasileiras: início precário, presente inebriante e o futuro, promissor?. Ciência Florestal, v. 24, n. 3, p. 771-784, 2014.

RIZZINI, C.T. Árvores e madeiras úteis no Brasil: manual de dendrologia brasileira. 1.ed. São Paulo: Blücher.1971. 292p.

ROBBRECHT, E. Tropical woody Rubiaceae. 1.ed. Bélgica: Opera Botanica Belgica, 1988., 271p.

SOTELO-MONTES, C. et al. Genetic variation in wood shrinkage and its correlations with tree growth and wood density of Calycophyllum spruceanum at an early age in the Peruvian Amazon. Canadian Journal of Forest Research, v. 37, n. 5, p. 966-976, 2007.

TAUCHEN, J. et al. Morphological and genetic diversity of Calycophyllum spruceanum (BENTH) K. SCHUM (Rubiaceae) in peruvian amazon. Agricultura Tropica et Subtropica, [s.n.], v. 44, p. 4, 2011.

TAVARES W.S. et al. Screening of extracts of leaves and stems of Psychotria spp. (Rubiaceae) against Sitophilus zeamais (Coleoptera: Curculionidae) and Spodoptera frugiperda (Lepidoptera: Noctuidae) for maize protection. Journal of Food Protection, n.11, p. 1892-1901, 2013.

VALLEJOS-TORRES, G. et al. Enraizamiento de brotes de capirona Calycophyllum spruceanum (Benth.) Hook. f. ex Schum., en la amazonía peruana. Revista Forestal Mesoamericana, v. 11, n. 27, p. 55-59, 2014.

WEBER, J. C.; SOTELO MONTES, C. Variation and correlations among stem growth and wood traits of Calycophyllum spruceanum Benth. from the Peruvian Amazon. Silvae Genet, v. 54, n. 1, p. 31-41, 2005.

UGARTE-GUERRA, L.J.; DOMÍNGUEZ-TORREJÓN, G. Índice de Sitio (IS) de Calycophyllum spruceanum Benth. en relación con la altura dominante del rodal en ensayos de plantación en la Cuenca del Aguaytía, Ucayali, Perú. Ecología Aplicada, v. 9, n. 2, p. 101111, 2010.

WEBER, J.C. et al. Phenotypic selection of Calycophyllum spruceanum on farms in the Peruvian Amazon: evaluating a low-intensity selection strategy. Silvae Genetica, v. 58, n. 4, p. 172, 2009.

YOUNG, M.C.M. et al. Chemosystematic markers of Rubiaceae. Opera Botanica Belgica, v. 7, [s.n.], p. 205-212, 1996.

ZULETA, L.M.C. et al. Seco-Iridoids from Calycophyllum spruceanum (Rubiaceae). Phytochemistry, v. 64, n.2, p. 549-553, 2003.

RUSSELL, J. R. et al. Genetic variation of Calycophyllum spruceanum in the Peruvian Amazon Basin, revealed by amplified fragment length polymorphism (AFLP) analysis. Molecular Ecology, v. 8, n. 2, p. 199-204, 1999.

Rev. Bras. PI. Med., Campinas, v.18, n.1, supl. I, p.383-389, 2016. 